dazu bereit wären, könnten wir diese Frage jetzt auf der Stelle regeln. Wir sind bereit, unsere Streitkräfte aus Deutschland abzuziehen, freilich unter der Bedingung, dass unsere Partner im Krieg gegen Hitlerdeutschland ihre Truppen abziehen.

Wir haben die Hoffnung auf Regelung dieser Frage in der Zukunft. Die Genfer Konferenz der Regierungschefs der vier Mächte hat diese Hoffnung geweckt. Wir hoffen, dass sich der Geist von Genf entwickeln und festigen wird, aber nicht als unsichtbarer und nicht wahrzunehmender Geist, sondern in Form konkreter Taten, wie dies von unserer Seite der Fall gewesen ist, als wir unsere Streitkräfte um 640000 Mann reduziert haben, 12 als wir auf die Verpachtung des Marinestützpunkts Porkkala-Udd in Finnland verzichtet haben ${ }^{13}$ und eine Reihe weiterer Maßnahmen durchgeführt haben. Wir warten darauf, dass unsere Partner in Genf in gleicher Weise reagieren, woraufhin wir unsererseits neue Vorschläge unterbreiten könnten.

Manche bürgerlichen Politiker warfen uns vor, dass wir zwar lächelten und uns freundschaftlich zeigten, jedoch angeblich nicht darüber hinaus gegangen sind. Sind denn die von mir angeführten Tatsachen der Streitkräftereduzierung und des Verzichts - sind das denn etwa keine konkreten Taten, die auf eine Abmilderung der internationalen Spannungen abzielen?

Gleichzeitig warten wir auf die konkreten Ergebnisse des Geistes von Genf aufseiten der Westmächte, die zur Abmilderung der internationalen Spannungen und zur Festigung der Sache des Friedens beitragen würden.

Übersetzt aus dem russischen Originaltext

RGANI, fond 52, opis' 1 , delo 557, Bl. 1-10

\title{
2. Gespräch Chruschtschows mit dem Vorsitzenden der Sozialistischen Partei Italiens, Pietro Nenni, am 15. Oktober 1955
}

Nach kurzen Ausführungen Chruschtschows über die UdSSR und die anderen sozialistischen Staaten verlief das Gespräch mit Pietro Nenni ${ }^{14}$ nach Aussage des sowjetischen Protokolls wie folgt.

Genosse Chruschtschow beleuchtete ausführlich die wichtigsten Maßnahmen der Sowjetunion zur Entspannung der internationalen Lage ${ }^{15}$ und ihre grundlegende Bedeutung für die Festigung der Position der Verfechter des Friedens in der gan-

12 Nach offizieller Erklärung wurden das Personal der sowjetischen Streitkräfte 1955 in zwei Schritten um insgesamt 640000 Mann reduziert.

13 Bei der Erneuerung des Beistandsvertrages mit Finnland am 19. September 1955 erklärte sich die UdSSR zur Räumung dieses Stützpunktes bereit, der im Friedensvertrag von 1947 für 50 Jahre an sie verpachtet worden war.

14 Nennis Sozialistische Partei stand - anders als die schwächeren, westlich orientierten Sozialdemokraten von Giuseppe Saragat - auf der Seite der UdSSR.

15 Chruščëv hatte im Kreml gegen den Widerstand von Außenminister Vjačeslav Molotov den Abschluss des Staatsvertrags mit Österreich am 15. Mai 1955 durchgesetzt, der das Ende der Besetzung durch die Vier Mächte und die Unabhängigkeit des Landes auf der Grundlage der von diesem erklärten Neutralität festlegte. Vom 26. Mai bis 2. Juni 1955 war er zusammen mit dem Vor- 
zen Welt. Die Präsenz der sowjetischen Truppen in Österreich wurde von den Feinden des Friedens stets als Beweis angeführt, dass die UdSSR einen Angriff vorbereite. Der Abschluss des Staatsvertrags mit Österreich aufgrund der Initiative der UdSSR und der Abzug der Besatzungstruppen wie auch der Verzicht der UdSSR auf Militärstützpunkte in China (Port Arthur) ${ }^{16}$ und Finnland (PorkkalaUdd $)^{17}$ hat unseren Feinden die Argumente genommen und die Position all derer gestärkt, die für den Frieden kämpfen. Durch die Übergabe von Porkkala-Udd an die Finnen wurden unlängst die Gründe für die Ängste auf Seiten der skandinavischen Länder - Dänemark, Norwegen und Schweden - beseitigt. Die bevorstehenden Reisen der Genossen Bulganin und Chruschtschow nach Indien und England sollen ebenfalls das gegenseitige Verständnis fördern und die internationalen Kontakte ausweiten.

Die französische Regierung hat unbedachterweise den Beschluss gefasst, die Reise von [Ministerpräsident] Faure und [Außenminister] Pinay in die UdSSR zu verschieben, offenbar unter dem Druck auswärtiger Kräfte. Jedoch hat Faure bereits erkennen lassen, dass der Gedanke an die Reise nicht begraben worden ist. ${ }^{18}$ Die sowjetisch-italienischen Beziehungen könnten viel enger sein. Unsererseits gibt es keine Hindernisse gegen die Ausweitung der gegenseitigen Beziehungen. Das Problem liegt auf der italienischen Seite, die möglicherweise aufgrund von Druck und Einmischung der Amerikaner in ihren Handlungen nicht frei ist.

Wir denken, sagte N. S. Chruschtschow, intensiv über die Einladung von Delegationen einiger sozialdemokratischer Parteien in die UdSSR nach, was das gegenseitige Verständnis fördern und die Friedenskräfte fördern könnte. Die Entscheidung, den Tourismus mit dem Ausland wieder aufzunehmen, hat bereits zu positiven Ergebnissen geführt. Visa für einen Aufenthalt in der UdSSR werden jetzt allen erteilt, die sich darum bemühen, sogar notorischen Reaktionären. Wir verweigern keinem ein Visum. Gemeinsam mit Gen. Bulganin, bemerkte Gen. Chruschtschow, haben wir eine Gruppe amerikanischer Kongressabgeordneter empfangen - [Senator Carey E.] Kefauver, [Senator George W.] Melone und andere - und ihnen erklärt, dass es an der Zeit ist, ihren „Eisernen Vorhang“ zu

sitzenden des Ministerrats der UdSSR, Nikolaj Bulganin, nach Belgrad gereist, um die 1948 durch den Konflikt Stalins mit Tito zerstörten Beziehungen zwischen den kommunistischen Parteien beider Länder wiederherzustellen. Die Genfer Gipfelkonferenz der Vier Mächte vom 18. bis 23. Juli rief in der Öffentlichkeit ein Gefühl der Entspannung zwischen Ost und West („Geist von Genf") hervor. Nachdem die sowjetische Regierung bereits am 25. Januar 1955 den Kriegszustand mit Deutschland für beendet erklärt hatte, lud sie am 7. Juni Bundeskanzler Adenauer nach Moskau zu Verhandlungen über die Aufnahme diplomatischer Beziehungen ein, die vom 9. bis 13. September stattfanden und zum Erfolg führten (vgl. u.a. Wettig, Sowjetische Deutschland-Politik 1953 bis 1958, München 2011, S. 46-58; Vizit kanclera Adenauera v Moskvu 8-14 sentjabrja 1955 g. Dokumenty i materialy. K 50-letiju diplomatičeskich otnošenij meždu Rossiej i Germaniej [Protokolle aus den Archiven der beiderseitigen Außenministerien], Moskau 2005).

16 Am 1.Juni 1955 hatte die UdSSR den 1945 in Besitz genommenen Marinestützpunkt Port Arthur (Lü-shun-kou) an China zurückgegeben.

17 Am 19. September 1955 hatte die UdSSR Finnland bei der Verlängerung des Freundschaftsvertrags von 1948 den Marinestützpunkt auf der direkt vor der Hauptstadt Helsinki gelegenen Halbinsel Porkkala zurückgegeben, dessen 50-jährige Verpachtung sie im Friedensvertrag von 1947 durchgesetzt hatte.

18 Der Besuch fand vom 15. bis 19. Mai 1956 statt, nachdem in Paris eine andere Regierung ihr Amt angetreten hatte. Daher verhandelte in Moskau dann nicht Edgar Faure, sondern der neue Ministerpräsident Guy Mollet (siehe die Dokumente Nr. 8 bis 13 in diesem Band). 
öffnen, da man zu ihnen, in die USA, nicht reisen kann. Sie haben keine Argumente für Einwände gefunden und zugegeben, dass die in den USA gültigen Gesetze überprüft werden müssen.

P. Nenni wurde über den sowjetischen Standpunkt zur Regelung der deutschen Frage informiert. ${ }^{19}$ Dabei teilte N. S. Chruschtschow Eindrücke von seiner DDR-Reise mit ${ }^{20}$ und gab eine Einschätzung der dortigen Situation und der dort erreichten Erfolge.

Hinsichtlich der Normalisierung der sowjetisch-jugoslawischen Beziehungen betonte N. S. Chruschtschow, dass die Verbesserung dieser Beziehungen nicht nur dem sowjetischen und dem jugoslawischen Volk großen Vorteil bringt, sondern sich auch als wichtiger Beitrag zur internationalen Entspannung erwiesen hat. Die Annäherung zwischen Jugoslawien und der UdSSR ${ }^{21}$ missfällt den regierenden Kreisen in den USA. Jetzt wird um die Seele des jugoslawischen Volkes gerungen. Wir sind von unserem Erfolg in diesem Wettstreit überzeugt. Zu den jugoslawischen Führern, besonders zu Tito, haben wir gute, freundschaftliche Beziehungen.

Gen. Mikojan hatte kürzlich bei einem Erholungsurlaub in Jugoslawien eine Reihe nützlicher Gespräche. Wir sind mit den Ergebnissen dieser Gespräche zufrieden.

Im Blick auf die Lage in den arabischen Ländern stellte N. S. Chruschtschow heraus, dass die Völker dieser Länder um ihre Selbständigkeit kämpfen und in Bewegung gekommen sind. Vor allem wird es von großer Bedeutung sein, dass Nasser dem Druck und der Erpressung standhält, die man im Zusammenhang mit den an ihn verkauften tschechoslowakischen Waffen, auf ihn ausübt. ${ }^{22}$ Die Imperialisten sind gewohnt, in diesen Ländern das Kommando zu führen ohne Rücksicht auf die Erwartungen der Völker, und sind empört, wenn diese Völker nicht mehr so leben wollen wie früher.

Nachdem Gen. Nenni, ${ }^{23}$ sagte Gen. Chruschtschow, kürzlich in China gewesen sei, kenne er die Erfolge der chinesischen Genossen. In den europäischen Ländern der Volksdemokratien stehen die Dinge gut, der Sozialismus siegt, ob-

19 Nach der 1949 festgelegten sowjetischen Auffassung war die Vereinigung Deutschlands Sache nur der „Deutschen selbst“, das heißt beider Staaten. Keine auswärtige Macht dürfe sich einmischen. Demnach konnte die staatliche Einheit allein durch Verhandlungen zwischen DDR und Bundesrepublik herbeigeführt werden. Das sollte von vornherein verhindern, dass die Westmächte das Thema im Zusammenhang mit dem Friedensvertrag zur Diskussion stellten.

20 Chruščëv versicherte der SED-Führung bei einem Zwischenaufenthalt in Ost-Berlin nach der Gipfelkonferenz in Genf vom 18. bis 23. Juli 1955, dass die UdSSR an ihrem Standpunkt in der deutschen Frage unverändert festhalte und die DDR voll unterstütze.

21 Vom 26. Mai bis 2. Juni 1955 waren Chruščëv und Bulganin in Belgrad und suchten den Bruch zwischen den kommunistischen Mächten Sowjetunion und Jugoslawien rückgängig zu machen, den Stalin durch seinen Bannstrahl gegen Tito 1948 herbeigeführt hatte. Beide Seiten verständigten sich zwar, doch zeigte sich bald, dass Tito nicht in das sowjetische Lager zurückkehrte, sondern nur von der Annäherung an den Westen abrückte. In der Blockfreienbewegung war er als einer der führenden Politiker aktiv.

22 Am 12. September 1955 hatten sowjetische und ägyptische Unterhändler in Prag die Lieferung moderner Waffensysteme aus der UdSSR an Ägypten vereinbart. Als dies wenig später der Regierung in Washington bekannt geworden war, reagierte diese heftig, weil sie darin eine Bedrohung Israels sah.

23 Nenni wird Genosse genannt, weil er mit seiner Sozialistischen Partei den Kommunisten eng verbunden war. 
wohl es noch Mängel gibt, die sowohl in der UdSSR als auch in jedem anderen Staat bestehen. Die Ideen des Sozialismus erfassen breite Bevölkerungsschichten aller Länder der Welt und sogar dort, wo die Arbeiterklasse noch nicht gesiegt hat, zieht man es vor zu sagen, dass man den sozialistischen Entwicklungsweg beschreite - in Indien, Indonesien, Burma usw. Insgesamt sind die Kräfte des Friedenslagers, der Demokratie und des Sozialismus den Kräften der Kriegsbefürworter überlegen. Generell geht es um unsere Fähigkeit, unsere Kräfte zu vereinigen, sie in Bewegung zu setzen, die riesigen Möglichkeiten und Reserven geschickt zu nutzen und sich nicht einschüchtern zu lassen. Im Westen gibt es große Meister der Angstmache und Einschüchterung, doch sie selbst haben Angst vor den Folgen eines Konflikts, weil sie wissen, was ihnen dann droht.

Nach aufmerksamem Zuhören dankte P. Nenni Gen. Chruschtschow warm und erklärte, dass er alles, was über die innere Lage der Sowjetunion gesagt worden sei, als sehr wertvoll zur Kenntnis nimmt, und mit der Einschätzung der internationalen Lage durch Gen. Chruschtschow voll übereinstimmt. Hinsichtlich der Situation in Italien bemerkte er, dass vielleicht noch mehr als die Amerikaner auf den Kurs der herrschenden italienischen Kreise die Tatsache einwirkt, dass dort im Unterschied zu allen anderen Ländern Westeuropas die linken Parteien - die Kommunisten und Sozialisten -eine gewaltige und geschlossene Kraft bilden. Aus Sorge, die Festigung dieser Parteien zu fördern, haben die italienische Bourgeoisie und die Regierung Angst vor einer Annäherung an die UdSSR und die Länder der Volksdemokratien. Das zeigt sich besonders deutlich, wenn es um die Aufnahme von Beziehungen zur ChVR24 [Chinesischen Volksrepublik] geht. Die italienischen Geschäftsleute wollen mit China Handel treiben, sie sehen die Vorteile eines derartigen Handels, und trotzdem ist die italienische Regierung abgeneigt und ergreift keine Maßnahmen. Übrigens ist diese Regierung nicht stabiler als das Kabinett von Faure in Frankreich.

Nach Ansicht Nennis bietet gegenwärtig unter allen Ländern Westeuropas allein Italien sehr günstige Umstände zur Erreichung eines entscheidenden Wandels der politischen Lage zugunsten der demokratischen Kräfte. ${ }^{25}$ Dabei betonte Nenni, er habe keinen revolutionären Weg im Auge, sondern die Gewinnung eines günstigeren Kräfteverhältnisses im Parlament. Wir, die italienischen Sozialisten, sagte er, sind keine Sozialdemokraten und überschätzen die Bedeutung des Parlamentes nicht. Gegenwärtig jedoch dreht und entwickelt sich der wesentliche politische Kampf im Land um den Einfluss auf die Wählermasse.

Die Entwicklung des Kampfes in Italien, fuhr Nenni fort, hängt vermutlich mehr als in jedem anderen Land von der internationalen Lage ab. Unsere Erfolge, die Erfolge der Kommunisten und Sozialisten Italiens, sind hauptsächlich von der Initiative der Sowjetunion zur Entspannung der internationalen Lage bestimmt. Wenn der Entspannungsprozess weiter anhält, dann kann ich versichern,

24 Diese Abkürzung wurde in der UdSSR allgemein verwendet, obwohl es üblicherweise Volksrepublik China heißt.

25 Als „demokratische Kräfte“ bezeichneten Nenni und Chruščëv nur Personen, Gruppen und Staaten, die für die sozialistischen Vorstellungen der UdSSR eintraten. 
erklärte Nenni, dass unsere zwei Parteien in den nächsten zwei Jahren den Sieg im Land erringen werden.

Weiter wiederholte Nenni in denselben Ausdrücken, die er zuvor im Gespräch mit den Gen. Malenkow und Suslow verwendet hatte, seine Ansicht über die Stärke der Kommunistischen Partei und der Sozialistischen Partei und über die Art ihres wechselseitigen Verhältnisses. Ich nutze die Gelegenheit, unterstrich Nenni, um Gen. Chruschtschow zu versichern, dass, welche Gerüchte darüber auch auftauchen mögen, die Einheit unserer beiden Parteien auch künftig unzerstörbar bleibt.

Was die Entwicklung der italienisch-sowjetischen Beziehungen betrifft, fuhr Nenni fort, nutzen die Kräfte der Reaktion im Innern ein Argument wie die Verweigerung der Aufnahme Italiens in die UNO durch die UdSSR 26 weidlich gegen uns aus. Diese Frage ist für uns sehr aktuell geworden. Im Gespräch mit den Gen. Malenkow und Suslow in Moskau habe er mit Befriedigung von dem sowjetischen Plan erfahren, Italien als eines der sechs Länder, die nach dem Krieg einen Friedensvertrag unterschrieben haben, ${ }^{27}$ zur UNO zuzulassen.

N. S. Chruschtschow bemerkte, dass er über den Inhalt des Gesprächs von P. Nenni mit den Gen. Malenkow und Suslow unterrichtet ist, und brachte ihm gegenüber vertraulich seine Auffassung über die Chancen der Aufnahme Italiens in die UNO zum Ausdruck. Der Vorschlag, sechs Länder aufzunehmen - das sei unser Plan, aber die westlichen Mächte würden ihm derzeit kaum zustimmen. Sie schlügen die Aufnahme von 16 Ländern vor, die den Antrag früher eingereicht hätten, dazu zwei weitere Länder - Japan und Italien. Dieser Plan stelle für uns ein Problem hauptsächlich deswegen dar, weil sich die UdSSR formal noch im Kriegszustand mit Japan befindet. Wir diskutierten gegenwärtig diese Variante. Jedenfalls könne man hoffen, dass, wenn nicht jetzt, so doch in Zukunft Italien in die UNO aufgenommen werde.

Nach seiner Rückkehr nach Italien, fuhr Gen. Nenni fort, werde er im Parlament die unverzügliche Entsendung einer Parlamentsdelegation in die UdSSR fordern. Ich habe hier, sagte er, französische, belgische, luxemburgische und sogar japanische parlamentarische Delegationen gesehen. Italien könne und dürfe den Austausch von Delegationen nicht länger hinauszögern.

N. S. Chruschtschow stimmte zu. Der Austausch von Parlamentsdelegationen könne das wechselseitige Verstehen fördern. Wir sind bereit, sagte er, Ihre Delegation $\mathrm{zu}$ empfangen. Daraufhin wird eine sowjetische Parlamentsdelegation nach Italien fahren können.

26 Die von den Westmächten seit 1949 immer wieder beantragte Aufnahme Italiens in die Vereinten Nationen scheiterte stets am Veto der UdSSR im Sicherheitsrat. Die Haltung wurde bis Dezember 1955 aufrechterhalten.

271947 waren weiterhin Friedensverträge geschlossen worden mit Finnland, das im folgenden Jahrvertraglich an die UdSSR gebunden wurde, sowie mit Ungarn, Rumänien und Bulgarien im sowjetischen Machtbereich. 1952 hatten die USA ohne Beteiligung der UdSSR einen Friedensvertrag mit Japan vereinbart. 
Die Normalisierung der sowjetisch-jugoslawischen Beziehungen, ${ }^{28}$ sagte $P$. Nenni weiter, habe zu Anfang in Italien Unverständnis hervorgerufen, vor allem bei den Kommunisten von Triest. ${ }^{29}$ Später sei Klarheit geschaffen worden, und die Sozialistische Partei erkenne, wie groß der Nutzen sei, den das Ende der Feindschaft zwischen der UdSSR und Jugoslawien für den Frieden gebracht habe. Er, Nenni, sei schon in den ersten Jahren nach dem Krieg für eine Regelung der Triest-Frage unter Berücksichtigung der Interessen Italiens eingetreten. Darüber habe er schon bei seinem ersten Treffen mit Gen. Molotow gesprochen. Solange die Triest-Frage noch in Italien diskutiert werde, müsse sich die Sozialistische Partei dazu äußern und eine Position beziehen, die den Jugoslawen nicht gefallen könne. Die Sozialisten werden sich jedoch in Zukunft bemühen, dem Prozess der Rückkehr Jugoslawiens in das sozialistische Lager nicht zu schaden.

Im Zusammenhang damit äußerte P. Nenni folgende Bitte: Wenn die Annäherung an Jugoslawien bereits so weit fortgeschritten ist, dass sie einen solchen Schritt gestatte, wäre es wünschenswert, Tito um ein Ende der gegen die Kommunistische und die Sozialistische Partei gerichteten politischen Arbeit in Italien zu bitten. Vielleicht nur in Italien, erläuterte Nenni, hätten die Jugoslawen vor einigen Jahren eine politische Organisation mit den aus der Kommunistischen Partei ausgeschlossenen Renegaten Cucchi und Magnani gegründet, die eine subversive Tätigkeit innerhalb und außerhalb der Kommunistischen Partei und der Sozialistischen Partei entfalte.

P. Nenni hob hervor, der von Gen. Chruschtschow dargelegte sowjetische Standpunkt zur deutschen Frage mache Korrekturen am Standpunkt der Italienischen Sozialistischen Partei erforderlich. Er, Nenni, habe bis dahin auf der Neutralität Deutschlands bestanden und bei seinen öffentlichen Auftritten erklärt, die deutsche Frage müsse durch Verhandlungen zwischen den vier Mächten gelöst werden. Uns war immer klar, sagte Nenni, dass die Vereinigung Deutsch-

28 Die Nachfolger Stalins hatten zwar 1953 die 1948 abgebrochenen diplomatischen Beziehungen wieder aufgenommen, doch verhinderte ablehnende Haltung von Außenminister Molotov, dass auch die - für das Verhältnis zwischen kommunistischen Staaten entscheidenden - Parteibeziehungen wiederhergestellt wurden. Erst als Chruščëv seit dem Frühjahr 1955 die Leitlinien der Außenpolitik bestimmte, wurde dieser Widerstand überwunden. Im Juni kamen er und Bulganin in Belgrad mit Tito überein, die Beziehungen zwischen den Parteien ebenfalls wieder aufzunehmen.

29 Nach dem Zweiten Weltkrieg erhob Jugoslawien Anspruch auf die italienische Stadt Triest, die zunächst von Titos Partisanen besetzt worden war, dann aber von diesen auf Druck der großen Siegermächte geräumt worden war. Bis zum Bruch mit Stalin 1948 machte Tito, den die UdSSR im Blick auf die Reaktion der Westmächte bestenfalls halbherzig unterstützte, die Forderung aggressiv geltend. Im Pariser Friedensvertrag mit Italien 1947 wurde die Stadt zusammen mit dem nordwestlichen Teil Istriens zum Freien Territorium erklärt, das einen vom Sicherheitsrat der UNO zu ernennenden Gouverneur erhielt. Wichtiger war, dass die Zone A, die vor allem von Italienern bewohnte Stadt Triest mit ihrer nächsten Umgebung, einer britisch-amerikanischen Militärverwaltung unterstellt wurde und dass die Zone B, das Hinterland und der Nordwesten Istriens mit weithin slowenischer Bevölkerung unter jugoslawische Militärverwaltung kam. Seit 1948 war die Regierung in Belgrad 1948 wegen des Bruchs mit der UdSSR auf die Zusammenarbeit mit den Westmächten angewiesen. Daher war sie 1954 zum Abschluss des Londoner Abkommens bereit. Danach kamen die Zone A unter italienische und die Zone B unter jugoslawische Zivilverwaltung. Trotzdem schwelte der Konflikt weiter. Das erklärt, wieso man in Rom nicht glücklich war über die Stärkung, die Jugoslawien durch das wiederhergestellte Verhältnis zur UdSSR gewann. 
lands unmöglich ist, wenn Westdeutschland in der NATO bleibt. Jetzt stellen wir in Übereinstimmung mit der Position der UdSSR die Forderung in den Mittelpunkt unserer Propaganda, dass die Deutschen selbst eine Übereinkunft über die Vereinigung Deutschlands erzielen müssen. Nenni erkannte an, dass es in der jetzt entstandenen Situation am vernünftigsten scheint, die Frage so zu stellen. Im Zusammenhang damit interessierte ihn, ob die Westmächte über den Standpunkt der Sowjetunion informiert worden sind und ob sie im Zusammenhang mit der bevorstehenden Außenministerkonferenz in Genf die Sowjetunion der Abkehr von der vereinbarten Position beschuldigen werden.

N. S. Chruschtschow informierte Nenni detailliert darüber, wie die erwähnte Position der Sowjetunion den anderen Mächten schon auf der Genfer [Gipfel-] Konferenz $z^{30}$ und danach in den öffentlichen Reden der Gen. Bulganin und Chruschtschow in Genf und [Ost-]Berlin ${ }^{31}$ zur Kenntnis gebracht worden ist. Vertraulich, zu Nennis persönlicher Kenntnis wurden ihm einige Einzelheiten der Abschlusssitzungen der Genfer Konferenz sowie der Umstände mitgeteilt, unter denen die Tagesordnung der Konferenz der Außenminister ausgearbeitet und angenommen worden ist. Der sowjetische Standpunkt zur deutschen Frage, sagte Gen. Chruschtschow abschließend, ist völlig fristgerecht rechtlich fixiert worden, ${ }^{32}$ und unsere Partner kennen ihn sehr gut.

Auf die Frage von P. Nenni gab Gen. Chruschtschow der Ansicht Ausdruck, dass auf der Konferenz der Außenminister die Sowjetunion erneut auf der Schaffung eines Systems der kollektiven Sicherheit in Europa bestehen werde. Im Zusammenhang damit legte N. S. Chruschtschow die sowjetischen Varianten eines solchen Systems dar, wie sie den Westmächten vorgeschlagen wurden. Das Fehlen einer einheitlichen Haltung aufseiten der Westmächte zur Frage der Sicherheit und Abrüstung wurde hervorgehoben. Am wahrscheinlichsten seien auf der bevorstehenden Konferenz einige Erfolge bei der Frage der Kontakte, selbstverständlich nur dann, wenn die westlichen Länder Interesse dafür zeigen.

N. S. Chruschtschow äußerte die Ansicht, nach der Aufnahme diplomatischer Beziehungen zwischen der UdSSR und der Deutschen Bundesrepublik ${ }^{33}$ würden die Deutschen mehr Neigung zu einer selbständigen Politik zeigen. In der deutschen Arbeiterklasse würden die für eine Annäherung an die DDR und den Schutz ihrer Errungenschaften eintretenden Kräfte an Stärke zunehmen.

30 Vom 18. bis 23. Juli 1955 trafen sich die Staats- und Regierungschefs der Vier Mächte in Genf zu einer Konferenz, in der es um eine Entspannung der Ost-West-Beziehungen ging, vor allem hinsichtlich der deutschen Frage und des Abrüstungsproblems.

31 Vgl. Dokumente zur Deutschlandpolitik, III. Reihe, Band 2/2, Bonn - [West-]Berlin 1963, S. 609621.

32 Im Freundschaftsvertrag vom 20. September 1955 billigte die UdSSR der DDR volle Souveränität $\mathrm{zu}$, behielt sich aber - so wie analog die Westmächte im Verhältnis zur Bundesrepublik - die Zuständigkeit für Berlin und für Deutschland als Ganzes vor.

33 Diese nicht korrekte Bezeichnung wurde in Moskau und Ost-Berlin oft statt des amtlichen Staatsnamens „Bundesrepublik Deutschland“ verwendet, um in Abwehr des westdeutschen Anspruchs auf allein demokratisch legitime Vertretung der Nation deutlich zu machen, dass die Bundesrepublik nicht das ganze Deutschland sei, sondern nur ein Teil. 
Im Blick auf das allgemeine Problem von Krieg und Frieden betonte Gen. Chruschtschow, dass der Deutschland-Frage das Allerwichtigste ist. Die Amerikaner würden nicht von sich aus Krieg führen. Auch England und Frankreich würden wohl kaum einen Krieg beginnen. Wenn sie das deutsche Volk nicht für ihre aggressiven Ziele benutzen könnten, würde es keinen Krieg in Europa geben. Die Westmächte versuchten mit ihrer NATO Einschüchterung zu betreiben, doch sei diese gar nicht so schreckenerregend, wie man sie hinstelle.

Zum Abschluss gab N. S. Chruschtschow seinen Vorstellungen über die Aufgaben des Kampfes für den Frieden Ausdruck und betonte, eine unermüdliche Wachsamkeit gegenüber den aggressiv gestimmten Kreisen im Westen sei notwendig. Diese Kreise versuchten die Ergebnisse der Genfer Konferenz ${ }^{34}$ zu verwischen. Einige Generäle träten wieder mit gehässigen Reden hervor, so wie jener kanadische General, der drohte, „die UdSSR kurz und klein zu schlagen“. Scherzhaft erzählte N. S. Chruschtschow, dass er ein Gespräch über diesen General mit [dem kanadischen Premierminister Lester] Pearson hatte und dass dieser den General einen törichten Menschen nannte.

N. S. Chruschtschow erklärte, die Macht des sozialistischen Lagers und der Verfechter des Friedens sei gewaltig und sei größer als die Macht der Kriegstreiber. Man muss diese Macht nur richtig und überlegt nutzen, ihre Mobilisierung stets aufrechterhalten und sich nicht einschüchtern lassen. Unter Bekundung der festen Zuversicht, dass die Sache des Friedens und des Sozialismus schließlich siegen wird, wünschte Gen. Chruschtschow Pietro Nenni und der Italienischen Sozialistischen Partei weitere Erfolge im gemeinsamen Kampf zusammen mit der Kommunistischen Partei für den Frieden und für die Lebensinteressen des italienischen Volkes.

Bei einem Spaziergang am Meer teilte N. S. Chruschtschow P. Nenni seine Beobachtungen mit hinsichtlich des Wachstums der Kolchosen, die er persönlich in Augenschein nimmt, und legte dar, wie das wichtigste Problem der Landwirtschaft gelöst wird: das Problem der Kader. Dazu sagte Nenni, nach seiner Ansicht stelle sich dieses Problem mit besonderer Schärfe für die chinesischen Genossen.

Als er sich von N.S. Chruschtschow verabschiedete, dankte Pietro Nenni ihm herzlich für den großartig gemeinsam verbrachten Tag und „für alles Bemerkenswerte, was ihm im Gespräch gesagt worden war“.

Nenni übergab Gen. Chruschtschow sein Buch „Vom Atlantikpakt zur Politik der Spannungsverringerung“, das in diesen Tagen auf russisch in Moskau erschienen war. In der Widmung bemerkte Nenni, aufgrund des Gesprächs habe sich

34 An die Genfer Gipfelkonferenz sprachen sich die Vier Mächte zwar prinzipiell für eine Verständigung in den Streitfragen aus. Es kam aber zu keiner Übereinkunft darüber, wie diese konkret zu erreichen sei. Darüber sollten die Außenminister auf ihrem Treffen im Oktober sprechen, doch der Dissens zwischen den Westmächten und der UdSSR ließ von vornherein keine Einigung erwarten. Die großen Erwartungen der westlichen Öffentlichkeit blieben unerfüllt. Trotzdem wurde in den sowjetischen Stellungnahmen weiter der „Geist von Genf“ beschworen in der Absicht, Entspannung und Einvernehmen als schon erreichte Errungenschaft hinzustellen, die den Aufbau eines Systems gemeinsamer kollektiver Sicherheit in Europa rechtfertige, das an die Stelle der gegeneinander gerichteten Bündnisse treten müsse. 
seine Zuversicht verstärkt, dass die Sache des Friedens und des Sozialismus den Sieg davontragen werde.

Das Gespräch dauerte 1 Stunde und 45 Minuten

Protokolliert von D. P. Schewljagin

Übersetzt aus dem russischen Originaltext

RGANI, fond 52, opis' 1 , delo 568, Bl.3-11

\section{Stellungnahme Chruschtschows bei Eröffnung der Tagung der kommunistischen und Arbeiterparteien der sozialistischen Länder am 6. Januar 1956}

Chruschtschow: Genossen! Ich denke, wir können mit der Arbeit beginnen. Gestattet mir einige Worte.

Im zurückliegenden Jahr 1955 haben die Sowjetunion, die Chinesische Volksrepublik, Polen, die Tschechoslowakei, Ungarn, die Deutsche Demokratische Republik, Rumänien, Bulgarien, Albanien und die anderen Länder des sozialistischen Lagers gewaltige Erfolge im Kampf um die Konsolidierung ihrer außenpolitischen Positionen und die Festigung des Friedens erzielt. In dieser Zeit hat sich die brüderliche Zusammenarbeit zwischen unseren Ländern weiter verstärkt.

Das sozialistische Lager ist zu einem wahren Bollwerk aller fortschrittlichen Kräfte der Welt geworden. Die Wirtschaft jedes der Staaten, die zu diesem Lager gehören, hat sich deutlich entwickelt und konsolidiert.

$\mathrm{Zu}$ den wichtigsten Errungenschaften des sozialistischen Lagers sind vor allem solche Ereignisse zu zählen wie der Friedensvertrag mit Österreich, ${ }^{35}$ die Beilegung des Konflikts mit Jugoslawien, ${ }^{36}$ die Aufnahme diplomatischer Beziehungen zwischen der UdSSR und der Deutschen Bundesrepublik, ${ }^{37}$ die Festigung der Beziehungen zwischen der UdSSR und Finnland dank der vorzeitigen Rückgabe des Militärstützpunkts Porkkala-Udd an die Finnen, ${ }^{38}$ die Reduzierung der Streitkräfte der Sowjetunion und Länder der Volksdemokratien. ${ }^{39}$ Eine große positive Rolle spielten auch die Besuche parlamentarischer Delegationen aus vielen

35 Am 15. Mai 1955 wurde der Staatsvertrag zwischen den vier Hauptsiegermächten des Zweiten Weltkriegs und Österreich geschlossen, der zwar die Funktion eines Friedensvertrags hatte, aber nicht als solcher bezeichnet wurde, weil Österreich nach amtlicher Lesart nicht zu den Kriegsgegnern gehört hatte, sondern als befreites Land anzusehen war.

36 Vom 26. Mai bis 2. Juni 1955 waren Chruščëv und Bulganin zur Wiederherstellung der 1948 abgebrochenen Parteibeziehungen in Belgrad.

37 In Verhandlungen vom 9. bis 13. September 1955 vereinbarte die sowjetische Führung mit Bundeskanzler Adenauer die Aufnahme diplomatischer Beziehungen.

38 Bei der Erneuerung des Beistandsvertrags mit Finnland am 19. September 1955 war die UdSSR zur Aufgabe des an die finnische Hauptstadt angrenzenden Marinestützpunkts Porkkala-Udd bereit, den sie gemäß dem Friedensvertrag von 1947 für 50 Jahre gepachtet hatte.

39 Die UdSSR fasste 1955 zwei Beschlüsse über die Reduzierung ihres Militärpersonals um insgesamt 640000 Mann. Im Zusammenhang damit konnten auch die mit ihr verbündeten Staaten ihre Truppenzahl vermindern. 